\title{
An upper bound for the chromatic number of line graphs
}

\author{
A. D. King ${ }^{\dagger}$ B. A. Reed ${ }^{\ddagger}$ and A. Vetta ${ }^{\S}$ \\ School of Computer Science, McGill University, 3480 University Ave., Montréal, Québec, H3A 2A7, Canada
}

It was conjectured by Reed [12] that for any graph $G$, the graph's chromatic number $\chi(G)$ is bounded above by $\left\lceil\frac{\Delta(G)+1+\omega(G)}{2}\right\rceil$, where $\Delta(G)$ and $\omega(G)$ are the maximum degree and clique number of $G$, respectively. In this paper we prove that this bound holds if $G$ is the line graph of a multigraph. The proof yields a polynomial time algorithm that takes a line graph $G$ and produces a colouring that achieves our bound.

\section{Introduction}

The chromatic number of a graph $G$, denoted by $\chi(G)$, is the minimum number of colours required to colour the vertex set of $G$ so that no two adjacent vertices are assigned the same colour. That is, the vertices of a given colour form a stable set. Determining the exact chromatic number of a graph efficiently is very difficult, and for this reason it has proven fruitful to explore the relationships between $\chi(G)$ and other invariants of $G$. The clique number of $G$, denoted by $\omega(G)$, is the largest set of mutually adjacent vertices in $G$ and the degree of a vertex $v$, written $\operatorname{deg}(v)$, is the number of vertices to which $v$ is adjacent; the maximum degree over all vertices in $G$ is denoted by $\Delta(G)$. It is easy to see that $\omega(G) \leq \chi(G) \leq \Delta(G)+1$. Brooks' Theorem (see [1]) tightens this:

Brooks' Theorem $\chi(G) \leq \Delta(G)$ unless $G$ contains a clique of size $\Delta(G)+1$ or $\Delta(G)=2$ and $G$ contains an odd cycle.

So for $\chi(G)$ we have a trivial upper bound in terms of $\Delta(G)$ and a trivial lower bound in terms of $\omega(G)$. We are interested in exploring upper bounds on $\chi(G)$ in terms of a convex combination of $\Delta(G)+1$ and $\omega(G)$. In [12], Reed conjectured a bound on the chromatic number of any graph $G$ :

Conjecture 1 For any graph $G, \chi(G) \leq\left\lceil\frac{\Delta(G)+1+\omega(G)}{2}\right\rceil$.

Several related results exist. In the same paper, Reed proved that the conjecture holds if $\Delta(G)$ is sufficiently large and $\omega(G)$ is sufficiently close to $\Delta(G)$. Using this, he proved that there exists a positive constant $\alpha$ such that $\chi(G) \leq \alpha(\omega(G))+(1-\alpha)(\Delta(G)+1)$ for all graphs. Some results are also known for generalizations of the chromatic number.

\footnotetext{
${ }^{\dagger}$ Corresponding author: king@cs.mcgill.ca. Research supported by NSERC and Tomlinson doctoral fellowships.

¥Research supported in part by a Canada Research Chair.

$\S$ Research supported in part by NSERC grant 28833-04 and FQRNT grant NC-98649.
} 
A fractional vertex c-colouring of a graph $G$ can be described as a set of stable sets $\left\{S_{1}, S_{2}, \ldots S_{l}\right\}$ with weights $\left\{w_{1}, w_{2}, \ldots w_{l}\right\}$ such that for every vertex $v, \sum_{S_{i}: v \in S_{i}} w_{i}=1$ and $\sum_{i=1}^{l} w_{i}=c$. The fractional chromatic number of $G$, written $\chi^{*}(G)$, is the smallest $c$ for which $G$ has a fractional vertex $c$-colouring. Note that it is always bounded above by the chromatic number. The list chromatic number of a graph $G$, written $\chi_{l}(G)$, is the smallest $r$ such that if each vertex is assigned any list of $r$ colours, the graph has a colouring in which every vertex is coloured with a colour on its list. For any graph we clearly have $\chi^{*}(G) \leq \chi(G) \leq \chi_{l}(G)$.

In [10], Molloy and Reed proved the fractional analogue of Conjecture 1 for all graphs, i.e. that

$$
\chi^{*}(G) \leq\left\lceil\frac{\Delta(G)+1+\omega(G)}{2}\right\rceil \text { for any graph } G .
$$

In fact, the round-up is not needed in the fractional case. In this paper we prove that Conjecture 1 holds for line graphs, which are defined in the next section.

\section{Fractional and Integer Colourings in Line Graphs of Multigraphs}

A multigraph is a graph in which multiple edges are permitted between any pair of vertices - all multigraphs in this paper are loopless. Given a multigraph $H=(V, E)$, the line graph of $H$, denoted by $L(H)$, is a graph with vertex set $E$; two vertices of $L(H)$ are adjacent if and only if their corresponding edges in $H$ share at least one endpoint. We say that $G$ is a line graph if there is a multigraph $H$ for which $G=L(H)$.

The chromatic index of $H$, written $\chi^{\prime}(H)$, is the chromatic number of $L(H)$. Similarly, the fractional chromatic index $\chi^{\prime *}(H)$ is equal to the fractional chromatic number of $L(H)$. In [6], Holyer proved that determining the chromatic index of an arbitrary multigraph is NP-complete, so practically speaking we are bound to the task of approximating the chromatic index of multigraphs and hence the chromatic number of line graphs.

Vizing's Theorem (see [14]) bounds the chromatic index of a multigraph in terms of its maximum degree, stating that $\Delta(H) \leq \chi^{\prime}(H) \leq \Delta(H)+d$, where $d$ is the maximum number of edges between any two vertices in $H$. Both bounds are achievable, but a more meaningful bound should consider other invariants of $H$. Of course, $\chi^{\prime}(H)$ is always bounded below by $\chi^{\prime *}(H)$, and Edmond's theorem for matching polytopes (presented in [3], also mentioned in [8]) tells us that given

$$
\begin{gathered}
\Gamma(H)=\max \left\{\frac{2|E(W)|}{|V(W)|-1}: W \subseteq H,|V(W)| \text { is odd }\right\}, \\
\chi^{\prime *}(H)=\max \{\Delta(H), \Gamma(H)\} .
\end{gathered}
$$

Does this necessarily translate into a good upper bound on the chromatic index of a multigraph? The following long-standing conjecture, proposed by Goldberg [4] and Seymour [13], implies that $\chi^{\prime *}(H) \leq$ $\chi^{\prime}(H) \leq \chi^{\prime *}(H)+1$ :

Goldberg-Seymour Conjecture For a multigraph $H$ for which $\chi^{\prime}(H)>\Delta(H)+1, \chi^{\prime}(H)=\lceil\Gamma(H)\rceil$.

Asymptotic results are known: Kahn [7] proved that the fractional chromatic index asymptotically agrees with the integral chromatic index, i.e. that $\chi^{\prime}(H) \leq(1+o(1)) \chi^{\prime *}(H)$. This implies the GoldbergSeymour Conjecture asymptotically. He later proved that in fact, the fractional chromatic index asymptotically agrees with the list chromatic index [8]. 
Another result that supports the Goldberg-Seymour Conjecture is the following theorem:

Theorem 2 (Caprara and Rizzi [2]) For any multigraph $H, \chi^{\prime}(H) \leq \max \{\lfloor 1.1 \Delta(H)+0.7\rfloor,\lceil\Gamma(H)\rceil\}$.

This theorem is a slight improvement of an earlier result of Nishizeki and Kashiwagi [11], lowering the additive factor from 0.8 to 0.7 . Note that this implies the Goldberg-Seymour Conjecture for any multigraph $H$ with $\Delta(H) \leq 12$, since in this case we have $\lfloor 1.1 \Delta(H)+0.7\rfloor \leq \Delta(H)+1$.

\section{The Main Result}

We will now prove our main result:

Theorem 3 For any line graph $G, \chi(G) \leq\left\lceil\frac{\Delta(G)+1+\omega(G)}{2}\right\rceil$.

Consider a multigraph $H$ for which $G=L(H)$. The proof consists of two cases: the case where $\Delta(G)$ is large compared to $\Delta(H)$, and the case where $\Delta(G)$ is close to $\Delta(H)$. In both cases we use the fact that $\omega(G) \geq \Delta(H)$. The first case is given by the following lemma, which follows easily from Theorem 2 .

Lemma 4 If $G$ is the line graph of a multigraph $H$, and $\Delta(G) \geq \frac{3}{2} \Delta(H)-1$, then $\chi(G) \leq\left\lceil\frac{\Delta(G)+1+\omega(G)}{2}\right\rceil$.

\section{Proof of Theorem 3:}

Consider a counterexample $G=L(H)$ such that the theorem holds for every line graph on fewer vertices. We know that $\Delta(G)<\frac{3}{2} \Delta(H)-1$. Our approach is as follows: We find a maximal stable set $S \subset V(G)$ that has a vertex in every maximum clique in $G$, and let $G^{\prime}$ be the subgraph of $G$ induced on $V(G) \backslash S$. We can see that $\Delta\left(G^{\prime}\right) \leq \Delta(G)-1$ (since $S$ is maximal) and $\omega\left(G^{\prime}\right)=\omega(G)-1$, and that the theorem holds for $G^{\prime}$, as any induced subgraph of a line graph is clearly a line graph. So we know that $\chi\left(G^{\prime}\right) \leq\left[\frac{\Delta(G)+1+\omega(G)}{2}\right]-1$. We can now construct a proper $\chi\left(G^{\prime}\right)+1$-colouring of $V(G)$ by taking a proper $\chi\left(G^{\prime}\right)$-colouring of $G^{\prime}$ and letting $S$ be the final colour class, hence $\chi(G) \leq\left\lceil\frac{\Delta(G)+1+\omega(G)}{2}\right\rceil$, a contradiction.

It suffices, then, to show the existence of such a stable set $S$ in $G$. We actually need only find a stable set that hits all the maximum cliques of $G$, as we can extend any such stable set until it is maximal. We will do this in terms of a matching in $H$, i.e. a set of edges in $E(H)$, no two of which share an endpoint - a matching in $H$ exactly represents a stable set in $G$. We need some notation first. For a pair of vertices $u, v \in V(H)$, the multiplicity of $u v$ is the number of edges in $E(H)$ between $u$ and $v$; we denote it by $\mu(u, v)$. A triangle in $H$ is a set of three mutually adjacent vertices, and we denote the maximum number of edges of any triangle in $H$ by $\operatorname{tri}(H)$; the edges of a triangle are those edges in $E(H)$ joining the triangle's vertices. Note the following facts that relate invariants of $H$ and $G$ :

Fact $1 \Delta(G)=\max _{u v \in E(H)}\{\operatorname{deg}(u)+\operatorname{deg}(v)-\mu(u, v)-1\}$.

Fact $2 \omega(G)=\max \{\Delta(H), \operatorname{tri}(H)\}$.

We say that a matching hits a vertex $v$ if $v$ is an endpoint of an edge in the matching. We will find a maximal matching $M$ in $H$ which corresponds to a desired stable set because it hits every vertex of maximum degree in $H$ and contains an edge of every triangle with $\max \{\Delta(H), \operatorname{tri}(H)\}$ edges in $H$.

To this end, let $S_{\Delta}$ be the set of vertices of degree $\Delta(H)$ in $H$ and let $T$ be the set of triangles in $H$ that contain $\max \{\Delta(H), \operatorname{tri}(H)\}$ edges. It is instructive to consider how the elements of $T$ interact; we omit the straightforward proofs of these observations from this extended abstract. 
Observation 1 If two triangles of $T$ intersect in exactly the vertices a and $b$ then ab has multiplicity greater than $\Delta(H) / 2$.

Observation 2 If abc is a triangle of $T$ intersecting another triangle ade of $T$ in exactly the vertex a then $\mu(b, c)$ is greater than $\Delta(H) / 2$.

Observation 3 If there is an edge of $H$ joining two vertices a and $b$ of $S_{\Delta}$ then $\mu(a, b)>\Delta(H) / 2$.

Guided by these observations, we let $T^{\prime}$ be those triangles in $T$ that contain no pair of vertices of multiplicity $>\Delta(H) / 2$ and $S_{\Delta}^{\prime}$ be those elements of $S_{\Delta}$ which are in no pair of vertices of multiplicity greater than $\Delta(H) / 2$. We treat $T^{\prime} \cup S_{\Delta}^{\prime}$ and $\left(T \backslash T^{\prime}\right) \cup\left(S_{\Delta} \backslash S_{\Delta}^{\prime}\right)$ separately. A few more observations regarding $S_{\Delta}^{\prime}$ and $T^{\prime}$ will serve us well. Again, we omit the proofs.

Observation 4 For any $S \subseteq S_{\Delta}^{\prime},|N(S)| \geq|S|$.

Observation 5 If an edge ab in $H$ has exactly one endpoint in a triangle bcd of $T^{\prime}$, then the degree of a is less than $\Delta(H)$.

Observation 6 If an edge ab in $H$ has exactly one endpoint in a triangle bcd of $T^{\prime}$, then $\mu(a, b) \leq$ $\Delta(H) / 2$.

Observation 7 For any vertex $v$ with two neighbours $u$ and $w, \operatorname{deg}(u)+\mu(v w) \leq \frac{3}{2} \Delta(H)$.

It is now straightforward to show that the desired matching exists. We begin with a matching $M$ consisting of one edge between each vertex pair with multiplicity greater than $\Delta(H) / 2$ - this hits $S_{\Delta} \backslash S_{\Delta}^{\prime}$ and contains an edge of each triangle in $T \backslash T^{\prime}$. Observation 4 tells us that we can apply Hall's Theorem (see [5]) to get a matching in $H$ that hits $S_{\Delta}^{\prime}$; Observation 7 dictates that this matching cannot hit $M$, so the union $M^{\prime}$ of these two matchings is a matching in $H$ that hits $S_{\Delta}$ and contains an edge of each triangle in $T \backslash T^{\prime}$. Every edge in this matching either hits a maximum-degree vertex in $H$ or has endpoints with multiplicity greater than $\Delta(H) / 2$.

What, then, can prevent us from extending this $M^{\prime}$ to contain an edge of every triangle in $T^{\prime}$ ? Observations 1 and 2 tell us that any two triangles in $T^{\prime}$ are vertex-disjoint, so our only worry is that $M^{\prime}$ hits two vertices of some triangle in $T^{\prime}$. Observations 3,5 and 6 guarantee that at most one such vertex in a given triangle is hit, and if there is such a vertex, it has degree $\Delta(H)$. We can therefore extend $M^{\prime}$ to contain an edge of every triangle in $T^{\prime}$. The result is a matching that satisfies all of our requirements, so the proof of the theorem is complete.

\section{Algorithmic Considerations}

We have presented a new upper bound for the chromatic number of line graphs, i.e. $\chi(G) \leq\left\lceil\frac{\Delta(G)+1+\omega(G)}{2}\right\rceil$. Our proof of the bound yields an algorithm for constructing a colour class in $G$ but we have an initial condition in the proof (i.e. $\Delta(G)<\frac{3}{2} \Delta(H)-1$ ) that does not necessarily remain if we remove these vertices. However, the bound given by Caprara and Rizzi in Theorem 2 can be achieved in $O(|E(H)|(|V(H)|+$ $\Delta(H)))$ time [2]. It is easy to see that in the proof of Theorem 3 we can find our matching in polynomial time, so we can formulate a polytime algorithm for $\left\lceil\frac{\Delta(G)+1+\omega(G)}{2}\right\rceil$-colouring a line graph $G$ with root graph $H$ as follows. 
1. While $\Delta(L(H))<\frac{3}{2} \Delta(H)-1$, remove a matching $M$ from $H$ as in the proof of Theorem 3 (and let it be a colour class).

2. Employ Caprara and Rizzi's algorithm to complete the edge colouring of $H$.

This, of course, assumes that we have the root graph $H$ such that $G=L(H)$. Lehot provides an $O(|E(G)|)$ algorithm that detects whether or not $G$ is the line graph of a simple graph $H$ and outputs $H$ if possible [9]. Two vertices $u$ and $v$ in $G$ are twins if they are adjacent and their neighbourhoods are otherwise identical. We can extend Lehot's algorithm to line graphs of multigraphs by contracting each set of $k$ mutually twin vertices in $G$ into a single vertex, which we say has multiplicity $k$. This can be done trivially in $O(|E(G)| \Delta(G))$ time. The resulting graph $G^{\prime}$ is the line graph of a simple graph $H^{\prime}$ if and only if $G$ is the line graph of a multigraph $H$; we can generate $H$ from $H^{\prime}$ by considering the multiplicities of the vertices in $G^{\prime}$ and duplicating edges in $H^{\prime}$ accordingly.

\section{References}

[1] R. L. Brooks. On colouring the nodes of a network. Proc. Cambridge Phil. Soc., 37:194-197, 1941.

[2] A. Caprara and R. Rizzi. Improving a family of approximation algorithms to edge color multigraphs. Information Processing Letters, 68:11-15, 1998.

[3] J. Edmonds. Maximum matching and a polyhedron with 0,1-vertices. Journal of Research of the National Bureau of Standards (B), 69:125-130, 1965.

[4] M. K. Goldberg. On multigraphs of almost maximal chromatic class. Diskret. Analiz, 23:3-7, 1973.

[5] P. Hall. On representation of subsets. J. Lond. Mat. Sc., 10:26-30, 1935.

[6] I. Holyer. The NP-completeness of edge-colouring. SIAM Journal on Computing, 10:718-720, 1981.

[7] J. Kahn. Asymptotics of the chromatic index for multigraphs. Journal of Combinatorial Theory Series A, 68:233-254, 1996.

[8] J. Kahn. Asymptotics of the list-chromatic index for multigraphs. Random Structures Algorithms, 17:117-156, 2000.

[9] P. G. H. Lehot. An optimal algorithm to detect a line-graph and output its root graph. J. Assoc. Comp. Mach., 21:569-575, 1974.

[10] M. Molloy and B. Reed. Graph Colouring and the Probabilistic Method. Springer-Verlag, Berlin, 2000.

[11] T. Nishizeki and K. Kashiwagi. On the 1.1 edge-coloring of multigraphs. SIAM Journal on Discrete Mathematics, 3:391-410, 1990.

[12] B. Reed. $\omega, \delta$, and $\chi$. Journal of Graph Theory, 27:177-212, 1998. 
[13] P. D. Seymour. Some unsolved problems on one-factorizations of graphs. In J. A. Bondy and U. S. R. Murty, editors, Graph Theory and Related Topics. Academic Press, New York, 1979.

[14] V. G. Vizing. On an estimate of the chromatic class of a p-graph. Diskret. Analiz, 3:23-30, 1964. In Russian. 